\title{
ANÁLISIS ECONÓMICO Y PRODUCCIÓN DEL MAÍZ (Zea mays L.) ASOCIADO CON MUCUNA (Stizolobium aterrimum) EN SIEMBRA DIRECTA Y DOS SISTEMAS DE FERTILIZACIÓN NITROGENADA
}

\author{
Betty González Osorio ${ }^{1}$ y Gorki Díaz Coronel ${ }^{2}$ \\ ${ }^{1}$ División Agrosocioeconómica, Unidad de Investigación Científica y Tecnológica, Facultad de Ciencias \\ Agrarias, Universidad Técnica Estatal de Quevedo. Dirección Via Sto. Domingo km 1 1/2 Av. 11 de Julio Teléfono, \\ Quevedo, Los Ríos, Ecuador. bettygon75@yahoo.com \\ ${ }^{2}$ Unidad de Investigación Cientifica y Tecnológica, Facultad de Ciencias Pecuarias, Universidad Técnica \\ Estatal de Quevedo. Quevedo, Los Ríos, Ecuador.
}

\section{RESUMEN}

Se realizó un estudio con el fin de conocer la rentabilidad y el efecto de la dosis de fertilización nitrogenada y del intercalado de $S$. aterrimum en el desarrollo y producción del híbrido de maíz INIAP H-551 bajo siembra directa en dos localidades. La dosis $200 \mathrm{~kg} \mathrm{ha}^{-1}$ de urea fraccionada en dos aplicaciones obtuvo el mejor desarrollo de planta, rendimiento del maíz y la mayor relación beneficio/costo. La mayor producción de biomasa se obtuvo con bajos niveles de N. A mayor cobertura de $S$. aterimun la incidencia de malezas fue menor. La nodulación presentó el mismo efecto a un menor contenido en el suelo de $\mathrm{N}$ y de $\mathrm{Ca}, \mathrm{Zn}, \mathrm{Fe}$ y Mo, la producción de nódulos aumenta. La dosis de $100 \mathrm{~kg} \mathrm{ha}^{-1}$ de urea fraccionada en dos aplicaciones presentó la mejor tasa marginal de retorno en términos físicos el punto de equilibrio fue de $3164.40 \mathrm{~kg}^{-1}$, observándose en ambos casos nuevas alternativas de producción para mejorar y recuperar suelos y la productividad del cultivo de maíz.

Palabras claves: Eficiencia agrícola, S. aterrimum, utilidad marginal.

\begin{abstract}
A study with the purpose of observing the profitability and the effect of the dose of nitrous fertilization and the inserted of S. aterrimum in the development and production of the INIAP H-551 hybrid corn planted in two places was carried out. It was found that the dose $200 \mathrm{~kg} \mathrm{ha}^{-1}$ of urea fractioned in two applications obtained the best plant development, yield of the corn and the biggest relationship cost/profit. When relating the production of biomass with the applied contents of the fertilization it was recorder that to smaller dose of $\mathrm{N}$ bigger covering of $\mathrm{S}$. aterrimun; and smaller overgrowth incidence; the nodulation presented the same effect to a smaller content in the soil of N, Ca, Zn, Fe and Mo, the production of nodules increases. The dose of $100 \mathrm{~kg}$ $\mathrm{ha}^{-1}$ of urea fractioned in two applications presented the best marginal rate of return in physical terms the balance point was at $3164.40 \mathrm{~kg} \mathrm{ha}^{-1}$, being observed in both cases new production alternatives to improve and to recover soils and the productivity of the corn cultivation.
\end{abstract}

Key words: Agricultural efficiency, S. aterrimum, marginal utility.

\section{INTRODUCCIÓN}

En la provincia de Los Ríos existen alrededor de 80914 ha de maíz, con una productividad que, en términos de rendimientos, fluctúa entre $1.5 \mathrm{tha}^{-1}$ (a nivel de los pequeños agricultores con tecnología tradicional) y $3.7 \mathrm{t} \mathrm{ha}^{-1}$ (a nivel tecnificado), en las mejores condiciones edafo-climáticas de la provincia (SICA, s.f.); las condiciones adecuadas, suelos propicios y un gran mercado de consumo han hecho que este cultivo se convierta en una de los rubros más importantes, para el pequeño productor de la provincia.

Sobre esta base, se planteó investigar si la siembra directa, uso de la mucuna ( $S$. aterrimum) alternada con el maíz combinado con la aplicación fraccionada de

Recibido: Octubre, 2007. Aceptado: Enero: 2008.

Publicado como ARTÍ́CULO en Ciencia y Tecnología 1: 37-41. 2008. dosis por hectárea de urea como fertilizante mejora la producción y la rentabilidad que percibe el productor. Se consideró que la siembra directa y la utilización de $S$. aterrimum como cultivo alternado con el maíz asegurarían la sustentabilidad de la producción por los beneficios que confiere al suelo por el no uso de la maquinaria agrícola, incremento de la materia orgánica y otros beneficios adicionales como el control de malezas (Díaz et al., 2004; Liebman, 1997) y erosión, etc. Por su parte, la aplicación fraccionada de la urea garantizaría un mejor aprovechamiento de este fertilizante nitrogenado (Crespo et al., 2003) y una mayor producción de maíz. De allí que, era importante realizar un análisis económico de todos estos factores para calcular su rentabilidad pues en cada uno de ellos se manifestarían los efectos sobre los costos del manejo del sistema de cultivo y de los ingresos por los rendimientos de los mismos. 
Dado lo anterior el objetivo de este estudio fue establecer la rentabilidad y el efecto de la fertilización nitrogenada y del intercalado de $S$. aterrimum en el desarrollo y producción del híbrido de maíz INIAP H-551 bajo siembra directa.

\section{MATERIALES Y MÉTODOS}

La investigación se realizó en dos sitios: la localidad 1 estuvo ubicado en el recinto El Pechiche, perteneciente al cantón Mocache y, la localidad 2 el otro, en el recinto San Luis, del cantón Quevedo, ambos sitios pertenecientes a la provincia de Los Ríos. Se utilizó un diseño de Bloques Completos al Azar, en arreglo factorial 2 (fertilizantes) x 2 (números de aplicaciones) y dos testigos (un testigo con urea diluida y otro sin urea), en cada localidad. Se dispuso de cuatro repeticiones por tratamiento. Se realizó un análisis combinado por localidad; todos los tratamientos llevaron maíz, híbrido INIAP-H 551 y S. aterrimum. Se realizó un análisis de suelo antes de la siembra y después de la cosecha, y se tomaron las siguientes mediciones en el maíz: altura y diámetro del tallo, longitud y diámetro de mazorca, peso de 1000 semillas, rendimiento por hectárea. En $S$ aterrimum: número y peso de nódulos, cobertura, producción de materia seca e incidencia de malezas. Para el análisis económico se utilizó el método de Perrin et al. (1979) el mismo que consistió en calcular un presupuesto parcial, análisis de dominancia y análisis marginal para obtener la Tasa Marginal de Retorno (TMR) y la Tasa Mínima de Retorno (TAMIR); además se calculó la estructura de costos, punto de equilibrio y eficiencia económica. Para estimar éste último indicador se tomó en consideración la máxima producción de maíz promedio de la provincia.

\section{RESULTADOS Y DISCUSIÓN}

La dosis de $200 \mathrm{~kg} \mathrm{ha}^{-1}$ de urea, fraccionada en dos aplicaciones se obtuvo la mejor respuesta en el crecimiento, diámetro de tallo y rendimiento de maíz, igual respuestas encontraron Crespo (2001); Amores y Mite (1993). Además, esta dosis y en dos aplicaciones de urea y asociado con $S$. aterrimum, mejoró el rendimiento de maíz, frente a la asociación de S. aterrimum sin aplicaciones de urea. La aplicación de urea granulada tuvo un efecto superior a la urea líquida debido a la mayor dosis aplicada con el fertilizante granulado, incidiendo positivamente la dosis más alta sobre el rendimiento (Figura 1). Comportamiento igual reportaron Estrada (1989); Menéndez et al. (2001) en estudios realizados en maíz con igual nivel de fertilización, en donde encontraron diferencias estadísticas altamente significativas.
La nodulación en la localidad 1 fue más vigorosa que la localidad 2, teniendo nódulos de hasta $2 \mathrm{~cm}$ de diámetro (Figura 2). La coloración de los nódulos en todos los tratamientos fue rojo y rosado pálido lo que indicó la presencia de la proteína lehemoglobina. La mejor nodulación estuvo asociada al menor contenido de nitrógeno y altos contenidos de $\mathrm{Ca}, \mathrm{Zn}, \mathrm{Fe}$ y Mo, lo cual incrementó el número y peso de los nódulos de $\mathrm{S}$. aterrimum, esto concuerda con lo reportado por Aranda et al. (2000); Altieri (1997); González y Lluch (1992); Mayea et al. (1998) (Cuadro 1).

La producción de biomasa de $S$. aterrimum estuvo directamente relacionada con el menor crecimiento del maíz, una menor fertilización, urea líquida o ausencia de urea como fuente de nitrógeno. La incidencia de malezas fue proporcional a la cobertura. A mayor cobertura de $S$. aterrimum menor incidencia de malezas. La presencia de hoja angosta fue superior a las de hoja ancha, predominando Euphorbia difusa y Cyperus esculentes. Los rubros más altos de los costos totales fueron: control de malezas, siembra y fertilización.

El maíz alternado con $S$. aterrimum con dosis de $100 \mathrm{~kg} \mathrm{ha}^{-1}$ de urea con dos aplicaciones se constituyó en la alternativa que presentó la mejor tasa marginal de retorno en las dos localidades bajo estudio (Cuadro 2). Y con la aplicación de $200 \mathrm{~kg} \mathrm{ha}^{-1}$ de urea fraccionado en dos aplicaciones se obtuvo la mejor relación beneficio/ costo (Cuadro 3). Por los mayores costos de producción de este tratamiento se considera que puede ser utilizado por los productores de mayores recursos y capacidad de riesgo. Este tratamiento presentó un índice de eficiencia agrícola de $1.25 \%$. El punto de equilibrio fue 3164.40 $\mathrm{kg} \mathrm{ha}^{-1}$ lo cual demuestra que es una nueva alternativa para el pequeño agricultor. (Figura 3).

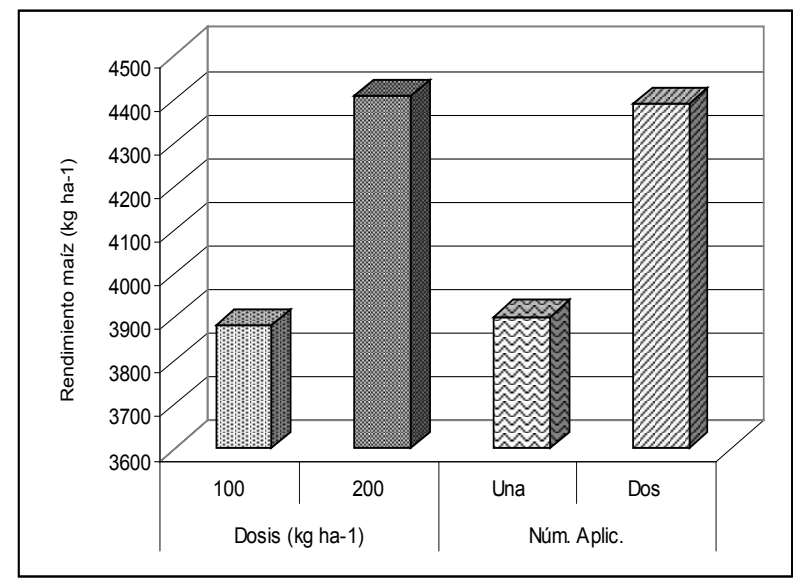

Figura 1. Efecto de la dosis y el número de aplicaciones de urea sobre el rendimiento del maíz 


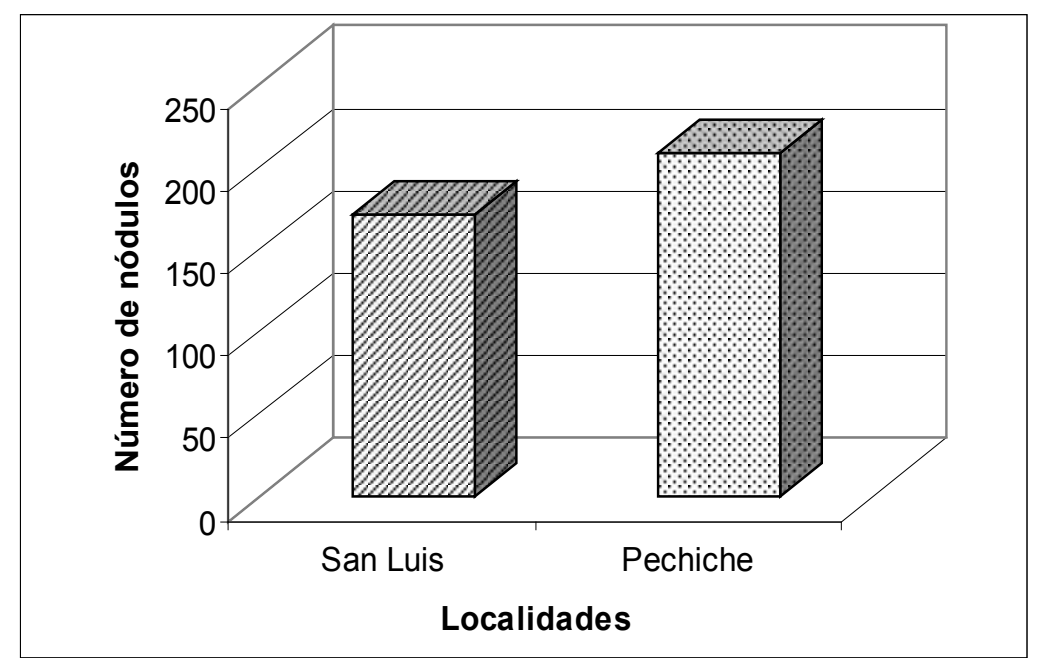

Figura 2. Número de nódulos de $S$. aterrimum en dos localidades de siembra de maíz

Cuadro 1. Análisis de suelo $(0-30 \mathrm{~cm})$ al inicio y final del ensayo de maíz alternado con $S$. aterrimum en dos localidades de siembra

\begin{tabular}{|c|c|c|c|c|c|c|c|c|}
\hline \multirow{4}{*}{$\begin{array}{l}\text { Nutrientes } \\
\mathrm{N}(\mathrm{ppm})\end{array}$} & \multicolumn{8}{|c|}{ Localidades } \\
\hline & \multicolumn{4}{|c|}{1} & \multicolumn{4}{|c|}{2} \\
\hline & \multicolumn{2}{|c|}{ Inicial } & \multicolumn{2}{|c|}{ Final } & \multicolumn{2}{|c|}{ Inicial } & \multicolumn{2}{|c|}{ Final } \\
\hline & 10 & $\mathrm{~B}^{*}$ & 13 & $\mathrm{~B}$ & 6 & B & 10 & $\mathrm{~B}$ \\
\hline $\mathrm{P}(\mathrm{ppm})$ & 34 & A & 35 & A & 9 & M & 11 & M \\
\hline $\mathrm{K}(\mathrm{meq} / 100 \mathrm{ml})$ & 0.58 & A & 0.58 & $\mathrm{~A}$ & 0.99 & A & 0.99 & A \\
\hline $\mathrm{Ca}(\mathrm{meq} / 100 \mathrm{ml})$ & 13 & A & 13 & $\mathrm{~A}$ & 14 & A & 14 & A \\
\hline $\mathrm{Mg}(\mathrm{meq} / 100 \mathrm{ml})$ & 1.5 & B & 2.8 & B & 2.2 & M & 2.5 & M \\
\hline $\mathrm{Zn}(\mathrm{ppm})$ & 4.5 & $\mathrm{M}$ & 4.8 & M & 10.7 & A & 10.9 & A \\
\hline $\mathrm{Cu}(\mathrm{ppm})$ & 8.5 & A & 8.5 & A & 20.1 & A & 20.1 & A \\
\hline $\mathrm{Fe}(\mathrm{ppm})$ & 121 & A & 121 & A & 209 & A & 209 & A \\
\hline $\mathrm{Mn}(\mathrm{ppm})$ & 3.4 & B & 3.5 & M & 44 & A & 43.7 & A \\
\hline $\mathrm{PH}$ & 6.1 & LA & 5.2 & A & 6.1 & MA & 5.3 & MA \\
\hline MO & 7.8 & A & 9.5 & A & 7.8 & A & 10.5 & A \\
\hline Arena & 48 & & 48 & & 35 & & 35 & \\
\hline Limo & 36 & & 36 & & 31 & & 31 & \\
\hline Arcilla & 16 & & 16 & & 34 & & 34 & \\
\hline
\end{tabular}

*A = Alto; M =Medio; B =Bajo; LA=Ligeramente Ácido; MA= Medianamente Ácido 
Cuadro 2. Análisis Marginal del promedio de seis tratamientos de dos localidades de siembra del maíz alternado con $S$. aterrimum

\begin{tabular}{cccccc}
\hline Tratamientos & $\begin{array}{c}\text { Costo } \\
\text { Variable }\end{array}$ & $\begin{array}{c}\text { Incremento } \\
\text { Marginal }\end{array}$ & $\begin{array}{c}\text { Beneficio } \\
\text { neto }\end{array}$ & $\begin{array}{c}\text { Incremento } \\
\text { marginal }\end{array}$ & $\begin{array}{c}\text { Tasa } \\
\text { marginal de } \\
\text { retorno }\end{array}$ \\
\hline $\begin{array}{c}100 \mathrm{~kg} \mathrm{ha}^{-1} \text { urea, } \\
\text { una aplic }\end{array}$ & 40 & 0 & 690.13 & 0 & \\
$\begin{array}{c}100 \mathrm{~kg} \mathrm{ha}^{-1} \text { urea, } \\
\text { dos aplic. }\end{array}$ & 50 & 10 & 761.06 & 70.93 & 709.30 \\
$\begin{array}{c}200 \mathrm{~kg} \mathrm{ha}^{-1} \text { urea, } \\
\text { dos aplic. }\end{array}$ & 80 & 30 & 850.33 & 89.27 & 297.57 \\
\hline
\end{tabular}

Cuadro 3. Promedio de costos (USD), ingresos (USD), rentabilidad y eficiencia agrícola por hectárea de maíz alternado con $S$. aterrimum y fertilización con urea granulada

\begin{tabular}{lcccccr}
\hline \multicolumn{1}{c}{ Rubro } & $\begin{array}{c}\mathbf{1 0 0 ~ k g ~ h a}^{-1} \\
\text { urea en una } \\
\text { aplicación }\end{array}$ & $\begin{array}{c}\mathbf{1 0 0 ~ k g ~ h a}^{-1} \\
\text { urea en dos } \\
\text { aplicaciones }\end{array}$ & $\begin{array}{c}\mathbf{2 0 0 ~ k g ~ h a}^{-1} \\
\text { urea en una } \\
\text { aplicación }\end{array}$ & $\begin{array}{c}\mathbf{2 0 0 ~ k g ~ h a}^{-1} \\
\text { urea en dos } \\
\text { aplicaciones }\end{array}$ & $\begin{array}{c}\text { Urea } \\
\text { líquida }\end{array}$ & Sin urea \\
\hline Costo total & 463.44 & 479.09 & 506.56 & 521.35 & 439.44 & 402.2 \\
Ingreso total & 730.13 & 811.06 & 818.82 & 930.33 & 498.61 & 384.45 \\
Rentabilidad & 0.58 & 0.69 & 0.62 & 0.78 & 0.13 & -0.04 \\
$\begin{array}{l}\text { Eficiencia } \\
\text { agrícola }\end{array}$ & 0.98 & 1.09 & 1.1 & 1.25 & 0.67 & 0.51 \\
\hline
\end{tabular}

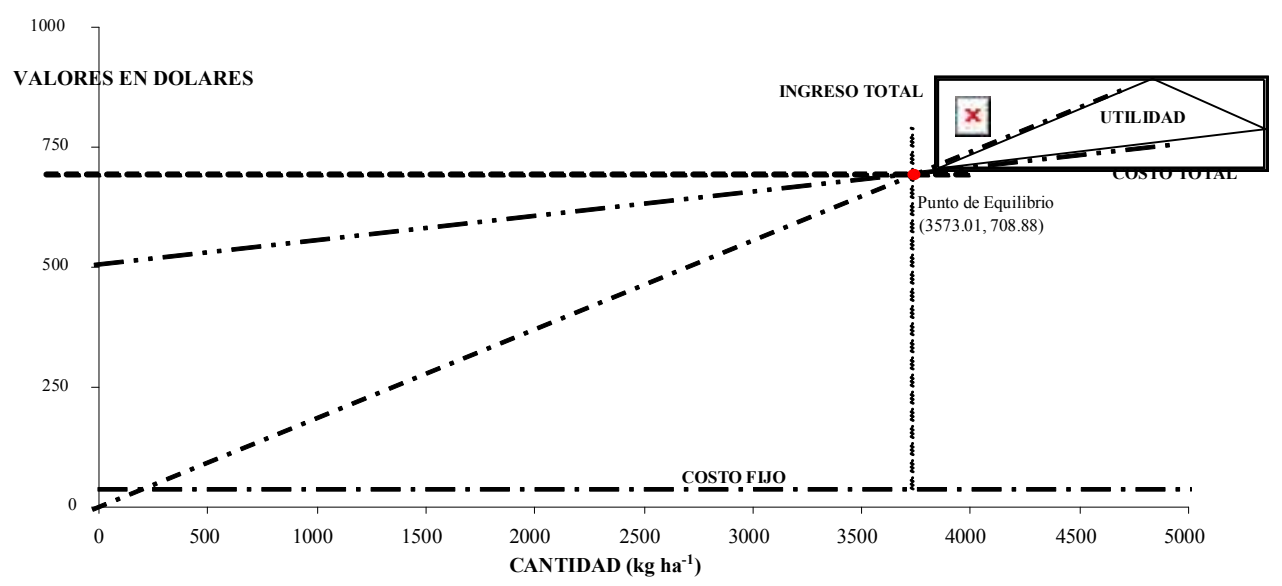

Figura 3. Puno de equilibrio el mejor tratamiento de maíz asociado con $S$. aterrimum en dos sitios de siembra

\section{CONCLUSIONES}

Con base en los resultados obtenidos en el presente estudio se concluye que la dosis $200 \mathrm{~kg} \mathrm{ha}^{-1} \mathrm{de}$ urea fraccionada en dos aplicaciones permitió obtener el mejor desarrollo de planta, rendimiento del maíz y la mayor relación beneficio/costo. La nodulación estuvo asociada a un menor contenido en el suelo de $\mathrm{N}$ y de $\mathrm{Ca}, \mathrm{Zn}, \mathrm{Fe}$ y Mo. La producción más alta de biomasa de $S$. aterrimum se dio cuando se aplicó menor dosis de N provocando esto mayor cobertura de $S$. aterrimum y logrando una menor incidencia de malezas. Con la dosis de $100 \mathrm{~kg} \mathrm{ha}^{-1}$ de urea fraccionada en dos aplicaciones se presentó la mejor tasa marginal de retorno en las dos localidades bajo estudio. En términos físicos el punto de equilibrio fue de $3164.40 \mathrm{~kg} \mathrm{ha}^{-1}$, por lo que se debe adoptar la siembra del maíz alternado con $S$. aterrimum en la 
dosis de $100 \mathrm{~kg} \mathrm{ha}^{-1}$ de urea fraccionada en dos aplicaciones para productores de escasos recursos y maíz alternado con $S$. aterrimum en la dosis de 200 $\mathrm{kg} \mathrm{ha}^{-1}$ de urea fraccionada en dos aplicaciones para productores de mayor solvencia económica.

\section{LITERATURA CITADA}

Altieri, C, M. 1997. Agroecología. Bases teóricas para una agricultura sustentable. Clades. Lima, Perú, 2 ed. p. 285-345.

Aranda, S; Hernandez, A; García, D y Simon, F. 2000(a). Aislamiento, caracterización y determinación de la actividad antagónica de cepas de rizobios ante Rhizoctonia solani. XII Seminario Científico, Programa y Resúmenes. 14 - 17 Noviembre. La Habana, CUBA. INCA 2000. $104 \mathrm{p}$.

Crespo, S; VIllavicencio, P y Valdivieso, C. 2003. INIAP H-552. Nuevo Híbrido de Maíz Amarillo Cristalino para la Zona Central del Litoral, Programa de maíz, E.E.T. Pichilingue, INIAP - PROMSA. Plegable divulgativo No 197.

Diaz, G; Estupiñán, K; Mite V, F; Amores P, P; Mera H, G. 2004. Evaluación de la mucuna, urea, roca fosfórica y zeolita para el incremento de la producción y fertilidad del suelo de pequeños productores de maíz. Universidad Técnica Estatal de Quevedo. Programa de Modernización de los Servicios Agropecuarios. Boletín Técnico no. 10. Quevedo-Ecuador. $20 \mathrm{p}$.

Gonzalez, J y Lluch, C. 1992. Biología del nitrógeno. Interacción planta - microorganismo. Ed Rueda. Madrid, ES. 43 p.

Liebman. M. 1997. Sistemas alternativos de producción. In. Altieri. M. Agroecología. Bases científicas para una agricultura sustentable. 2 ed. Lima - Perú. p. 285-327.

Mayea, S; Coroneo, M; Novo, R; Boado, I; Silveira, E; Soria, M; Morales, Y. y Valiño, A. 1998. Microbiología agropecuaria. Tomo II Ed. Félix Varela. La Habana, CU. p $156-178$.

Meléndez, L. Lisazo, J. Ramírez, R. 2001. Efecto de la fertilización nitrogenada sobre dos variedades de maíz (Zea mays L.) sometidas a exceso de humedad en el suelo. Disponible en: http://www.fertilizar.org.ar/ articulosFertilizaciónNitrogenadadelcultivo.

Perrin, R.; Winkelmann, D.; Moscardi, E.; Anderson, J. 1979. Formulación de recomendaciones a partir de datos agronómicos. Un manual metodológico de evaluación económica, Centro Internacional de Mejoramiento de Maíz y Trigo. México. 4 p.

SICA (SERVICIO DE INFORMACIÓN Y CENSO AGROPECUARIO, EC). s.f. Ecuador: panorama de la cadena agroalimentaria.Consultado 13 sept. 2005. Disponible en http://www.sica.gov.ec/cadenas/maíz/ docs/panorama.cadena.html. 